Check for updates

Cite this: RSC Adv., 2017, 7, 26434

Received 7th April 2017

Accepted 11th May 2017

DOI: $10.1039 / \mathrm{c} 7 \mathrm{ra03979d}$

rsc.li/rsc-advances

\section{Linear magnetoresistance in gold foams}

\author{
Zhaoguo Li, iD* Jiangshan Luo, Xiulan Tan, Qi Fang, Yong Zeng, Lingbiao Meng, \\ Minjie Zhou, Weidong Wu and Jicheng Zhang*
}

The electrical transport properties of metal foams may not only be of fundamental research interest but can also lead to wide application in sensors. We report the magnetotransport properties of gold foams prepared by a chemical template-dealloying method. The temperature dependence of the resistivity of gold foams represents a metallic behavior with zero magnetic field. With increasing magnetic field, a crossover from quadratic to linear dependence of the magnetoresistance in gold foams is observed in the studied temperature range $(2-50 \mathrm{~K})$. The physical mechanisms resulting in this observation may be ascribed to the classical linear magnetoresistance theory based on the spatial mobility fluctuations or current distribution effect. However, further evidence is required to determine the exact mechanism.

\section{Introduction}

The electrical transport study of nanostructures has attracted substantial research interest in the past decades. Various novel physical phenomena have been discovered, such as the quantum Hall effect in quantum wells, ${ }^{1}$ the single-electron tunneling in quantum dots, ${ }^{2}$ the quantum interference effect in disordered systems, ${ }^{3}$ the giant magnetoresistance (MR) effect in alternating ferromagnetic and non-magnetic layered films, ${ }^{4}$ and so forth. The newly discovered artificial nanostructures have also promoted quantum transport studies, such as carbon nanotubes, ${ }^{5}$ graphene, ${ }^{6}$ topological insulators, ${ }^{7}$ transition metal dichalcogenides, ${ }^{8}$ and so forth. Overall, the transport study of nanostructures will remain a very active research area in the future owing to its importance in fundamental physics and for practical applications.

The low-density gold foam is a typical nanostructured material, which exhibits numerous potential applications in catalysts, sensors, plasmonics, and so forth, owing to the high surface-volume ratio and low electrical resistivity. ${ }^{9}$ The electrical transport study of gold foams is a critical issue for exploring the effect of the three-dimensional (3D) interior topology of nanostructures on their electrical transport properties. ${ }^{\mathbf{1 0 - 1 4}}$ Fujita et al. $^{\mathbf{1 0}}$ reported extremely low MR values for nanoporous gold films. Their work underscored the importance of the 3D topology of a nanostructure on the electronic transport properties. Xia et al. ${ }^{\mathbf{1 1}}$ observed that the thermal and electrical conductivities of nanoporous gold films were significantly lower than those of bulk gold, but the corresponding Lorenz number was strikingly similar to that of bulk gold. Their work reveals the anomalous electrical transport

Research Center of Laser Fusion, China Academy of Engineering Physics, Mianyang 621900, P. R. China.E-mail: lizhaoguoabc@163.com; zhangjccaep@126.com properties in nanoporous gold materials; however, the physical mechanisms of the above experimental phenomena remain unknown. Therefore, further experimental and theoretical efforts are required in this direction.

Here, we report on the magnetotransport behavior of gold foams. The temperature dependence of the resistivity of gold foams represents a metallic behavior with zero magnetic field. The MR curves between $-9 \mathrm{~T}$ and $9 \mathrm{~T}$ for gold foams are measured in the low temperature regime $(2-50 \mathrm{~K})$. A crossover from a quadratic to a linear dependence of the MR in gold foams is observed with an increase of the magnetic field. The physical mechanisms behind this observation are discussed.

\section{Methods}

The gold foams studied in this work were prepared by a chemical template-dealloying method. First, Au and Ag nanoparticles were electroless deposited onto polystyrene (PS) microspheres which were employed as the sacrificial template. Next, the cylindrical $\mathrm{PS} /(\mathrm{Au}-\mathrm{Ag})$ monolith was formed by filter-casting of the nanoparticle coated PS suspension. Then, the self-supported $\mathrm{Au}-\mathrm{Ag}$ foams were produced by removing the polystyrene template. The self-supported gold foams with bimodal porous structure were finally obtained after dealloying of Ag. The details of the preparation technology can be found in ref. 15. The structure of gold foams was characterized by X-ray diffraction (XRD), scanning electron microscopy (SEM) and X-ray computed tomography (CT). To measure the resistivity, the Hall-type electrodes were attached onto the gold foams by using silver paint. The magnetotransport measurements of the gold foams were performed on a Quantum Design physical property measurement system, which can provide a low temperature condition of $T=2 \mathrm{~K}$ in magnetic fields up to $B=9 \mathrm{~T}$. 
Table 1 Parameters of samples. $l$ is the conduction length (i.e., the closest distance between the two voltage probes), $w$ is the width, $t$ is the thickness, $\rho_{0}$ is the resistivity, $\mu$ is the electron mobility, $n$ is the electron concentration, $\Delta \rho(9 \mathrm{~T}) / \rho(0)=[\rho(B=9 \mathrm{~T})-\rho(0)] / \rho(0) \times 100 \%$. The values of $\rho_{0}, \mu, n$ and $\Delta \rho(9 \mathrm{~T}) / \rho(0)$ are for $2 \mathrm{~K}$

\begin{tabular}{|c|c|c|c|c|c|c|c|}
\hline Sample & $l(\mathrm{~mm})$ & $w(\mathrm{~mm})$ & $t(\mathrm{~mm})$ & $\rho_{0}(\mu \Omega \mathrm{m})$ & $\mu\left(\mathrm{cm}^{2} \mathrm{~V}^{-1} \mathrm{~s}^{-1}\right)$ & $n\left(\mathrm{~cm}^{-3}\right)$ & $\Delta \rho(9 \mathrm{~T}) / \rho(0)(\%)$ \\
\hline S1 & 1.0 & 0.5 & 0.3 & 1.41 & 6.2 & $4.13 \times 10^{21}$ & 1.35 \\
\hline S2 & 0.8 & 0.9 & 0.4 & 2.45 & 10.8 & $4.11 \times 10^{21}$ & 1.87 \\
\hline S3 & 1.1 & 0.8 & 0.6 & 2.01 & 9.7 & $3.21 \times 10^{21}$ & 1.50 \\
\hline Au film & 0.05 & 0.01 & $3 \times 10^{-5}$ & 0.012 & 69 & $7.45 \times 10^{22}$ & 0.39 \\
\hline
\end{tabular}

\section{Results}

We investigated three gold foams that were cleaved from a monolith. For comparison, a gold film prepared by electronbeam evaporation was also studied. The parameters of these samples are listed in Table 1.

Fig. 1(a) shows the SEM image of the gold foams. We observed that the foam consists of gold microspherical shells. The details of the single microspherical shell are shown in Fig. 1(b), where the diameter of the shells was approximately 10 $\mu \mathrm{m}$. The hollow structure of the gold microspherical shells can be identified by nano-CT measurements, as shown in Fig. 1(c), and the thickness of the shells was obtained as $\sim 400 \mathrm{~nm}$. The XRD measurements show the polycrystalline structure of the gold foams [Fig. 1(d)]. Fig. 1(e) shows the temperature dependence of the resistivity $\rho(T)$ for the three gold foam samples (named as S1, S2 and S3) at zero magnetic field. The electrical resistivity decreased with a decreasing temperature, which is a typical metallic behavior. The values of the gold foams' resistivity at low temperatures were in the order of $1 \mu \Omega \mathrm{m}$, which is 100 times larger than the resistivity of the gold film (Table 1). The large difference in resistivity between gold foams and films originated from the high porosity of the foams. The calculated resistivity of the gold foams, as indicated in Table 1, did not exclude the voids in the foams. This issue will be discussed further below.

Fig. 2(a-c) display the MR curves at $T=2 \mathrm{~K}$ for $\mathrm{S} 1$ to $\mathrm{S} 3$, respectively, where the MR value is defined as $\Delta \rho(B) / \rho(0)=[\rho(B)$ $-\rho(0)] / \rho(0) \times 100 \%$. A nonsaturating of the MR values at a very high magnetic field can be observed. The insets of Fig. 2(a) and (b) show the field derivative of $\mathrm{MR}$, that is, $\mathrm{d} \rho(B) / \mathrm{d} B$ of $\mathrm{S} 1$ and $\mathrm{S} 2$, respectively. We can see that $\mathrm{d} \rho / \mathrm{d} B$ initially increased linearly with an increase in the field, which indicates the $B^{2}$ dependence of $\rho(B)$. Then, $\mathrm{d} \rho / \mathrm{d} B$ was saturated for $B$ above the critical field
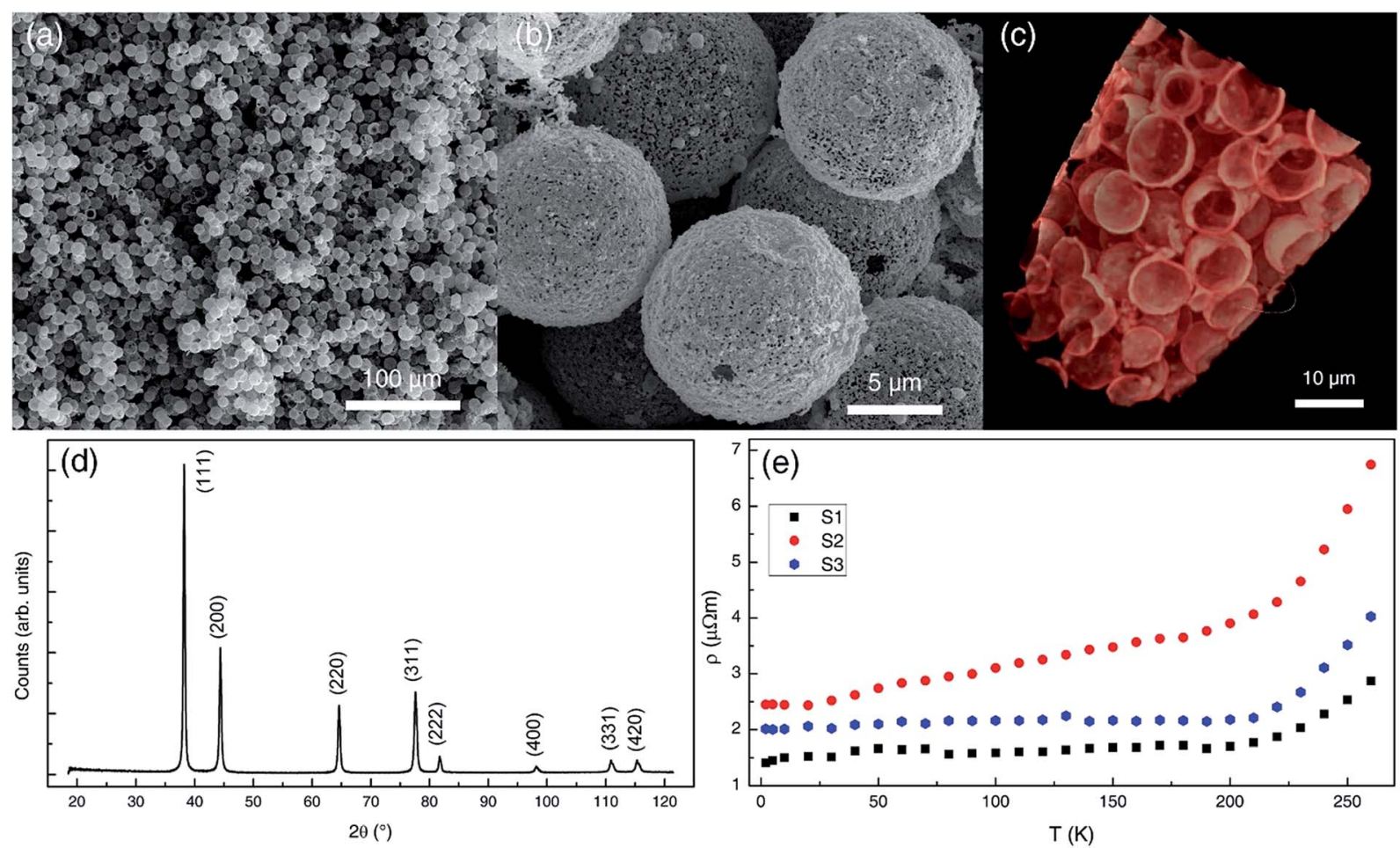

Fig. 1 Characterization of the gold foams. (a) Scanning electron microscopy (SEM) image of the gold foam monolith. (b) Enlarged SEM image of the hollow microspherical shells of gold foams. (c) Reconstructed three-dimensional structure of gold foams imaged by $\mathrm{X}$-ray computed tomography. (d) X-ray diffraction patterns of gold foams. The dominant diffraction peaks are labelled, which belong to the gold crystals. (e) Temperature dependence of resistivity of the three gold foam samples at zero magnetic field. 

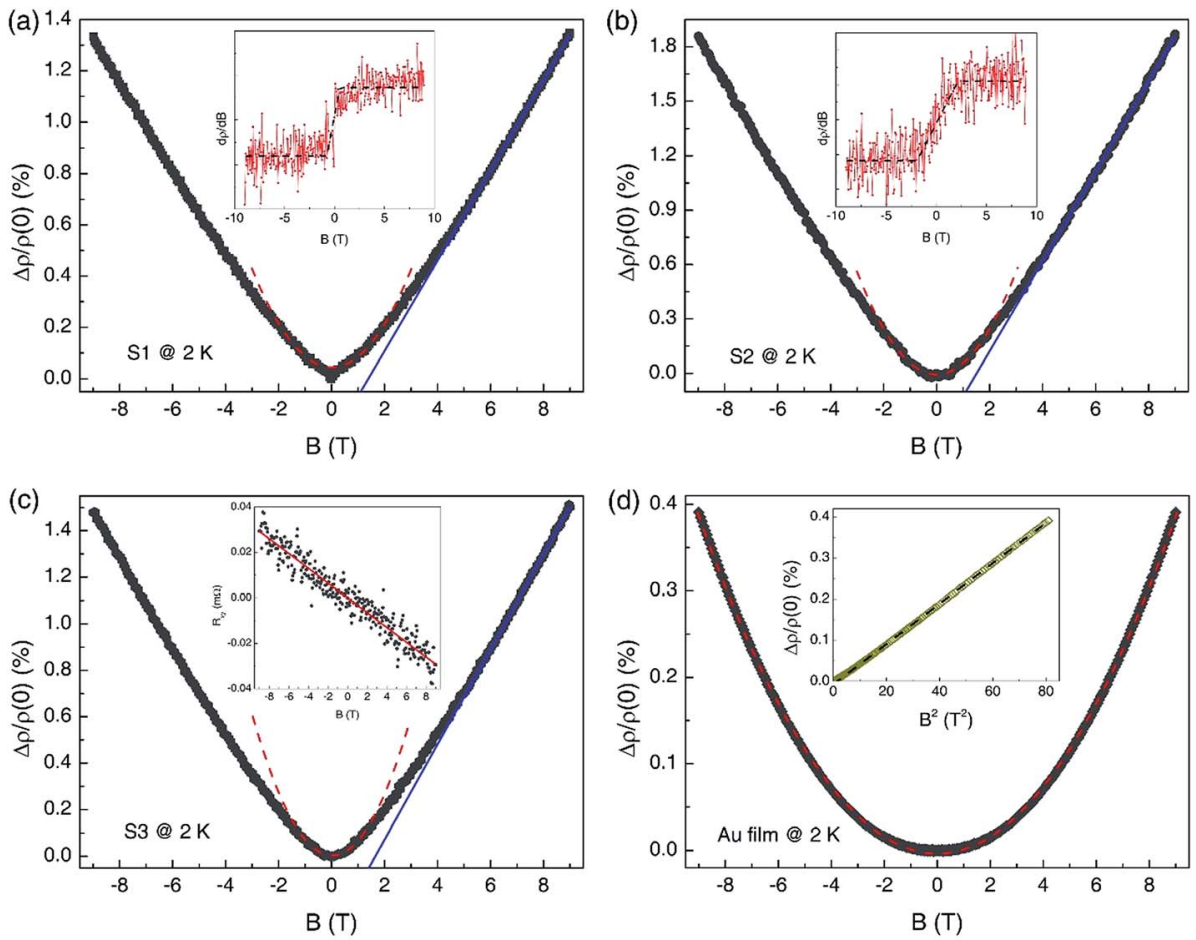

Fig. 2 Magnetoresistance (MR) of gold foams at $T=2 \mathrm{~K}$. MR curves of (a) foam samples S1, (b) S2 and (c) S3 in the perpendicular field $B$, where $\Delta \rho(B) / \rho(0)=[\rho(B)-\rho(0)] / \rho(0) \times 100 \%$. The solid lines are the linear fitting results of high field MR and are used as guides for the eye. The dashed curves are the Kohler fits to the low field MR. The insets of (a) and (b) show the $\mathrm{d} \rho(B) / \mathrm{d} B$ curves of S1 and S2, respectively, the dashed curves are guides for the eye. The inset of (c) shows the Hall resistance $R_{\mathrm{xy}}$ plotted as a function of field $B$ for $\mathrm{S} 3$. The solid line is the linear fitting of the $R_{\mathrm{xy}}-B$ data. (d) MR curve of a gold film, the dashed curve is the Kohler fit. The inset shows the $\Delta \rho(B) / \rho(0)$ plotted as a function of $B^{2}$, the dashed line is the linear fitting result as a guide for the eye.

[the dashed curves in the insets of Fig. 2(a) and (b)], which corresponds to a linear MR. The solid lines in Fig. 2(a-c) indicate the linear MR curves of the gold foams. Fig. 2(d) shows the MR curve of a homogeneous Au film at $T=2 \mathrm{~K}$. The $\Delta \rho(B) / \rho(0)$ plotted as a function of $B^{2}$ for the gold film is shown in the inset of Fig. 2(d). The linear dependence of $\Delta \rho(B) / \rho(0)-B^{2}$ revealed the classical magnetotransport in the gold films. The classical diffusion of electrons in materials arising from the Lorentz force will lead to a $B^{2}$ dependence of MR, that is, Kohler's rule: ${ }^{16}$ $\Delta \rho(B) / \rho(0) \simeq(\mu B)^{2}$, where $\mu$ is the electron mobility. The dashed curve in Fig. 2(d) is the Kohler fitting result. A good fitting result is obtained over the whole magnetic field range of the gold film. For the gold foams, we can see that Kohler's rule was valid only in the low-field regime $(|B| \lesssim 2 \mathrm{~T})$, and the high-field regime $(|B| \gtrsim 3 \mathrm{~T})$ was dominated by a linear MR behavior [Fig. 2(a-c)].

The inset of Fig. 2(c) shows the Hall resistance of sample S3. The linear fitting of the $R_{\mathrm{xy}}-B$ curve gives the electron concentration of the gold foams $n_{\text {foam }} \approx 3.21 \times 10^{21} \mathrm{~cm}^{-3}$ for S3. By using the free electron gas model, we could estimate the electron mobility in gold foams as $\mu=1 /($ ne $\rho) \approx 9.7 \mathrm{~cm}^{2} \mathrm{~V}^{-1} \mathrm{~s}^{-1}$ for S3. Additionally, $n_{\text {film }} \approx 7.45 \times 10^{22} \mathrm{~cm}^{-3}$ was also obtained, which is consistent with the values of bulk gold. ${ }^{17}$ The electron concentration $n$ and mobility $\mu$ of all samples are listed in Table 1 . We observe that the value of $n_{\text {film }}$ was $\sim 20$ times larger than that of $n_{\text {foam }}$. Intuitively, the reason for this could be ascribed to the voids in the foams as the calculated $\rho$ did not exclude the void volumes in the gold foams. If we consider the porosity of the gold foams $\left[\sim 80 \%\right.$ (ref. 15)], the revised $n_{\text {foam }}$ was in the order of $10^{22} \mathrm{~cm}^{-3}$. This value is consistent with the values of the gold films and bulk.

Fig. 3(a) and (b) represent the MR curves of gold foam samples S1 and S2, respectively, from $2 \mathrm{~K}$ to $50 \mathrm{~K}$. Several features of these MR curves were distinguishable, such as, the MR is (i) positive; (ii) relatively large $(\sim 1.9 \%$ at $B=9 \mathrm{~T}$ and $T=2$ $\mathrm{K}$ ); (iii) unsaturated in the high-field regime that is directly proportional to the magnetic field over the whole temperature range. Moreover, the MR curves with $T \lesssim 20 \mathrm{~K}$ were nearly coincident with each other. For temperatures exceeding $\sim 20 \mathrm{~K}$, the MR values were weakened, and the magnetic field range where Kohler's rule was dominant was widened. For example, at $T=50 \mathrm{~K}$, Kohler's rule was valid in the low-field regime for $|B|$ $\leq 5 \mathrm{~T}$, and a linear MR behavior could be observed in the highfield regime $(|B| \gtrsim 5 \mathrm{~T})$. Fig. $3(\mathrm{c})$ shows the MR values at $B=9 \mathrm{~T}$ plotted as a function of the temperature for the gold foam samples. Fig. 3(d) displays the temperature dependence of the electron mobility $\mu$ that was extracted from the Hall fitting of the $R_{\mathrm{xy}}-B$ data of the gold foams. We can see that $\Delta \rho(9 \mathrm{~T}) / \rho(0)$ and $\mu$ were nearly constant at $T \lesssim 20 \mathrm{~K}$, and they were decreased when the temperature exceeds $\sim 20 \mathrm{~K}$ owing to the enhancement of the thermal phonon scattering in the gold foams. These tendencies are consistent with the results from nanoporous gold. ${ }^{10}$ 

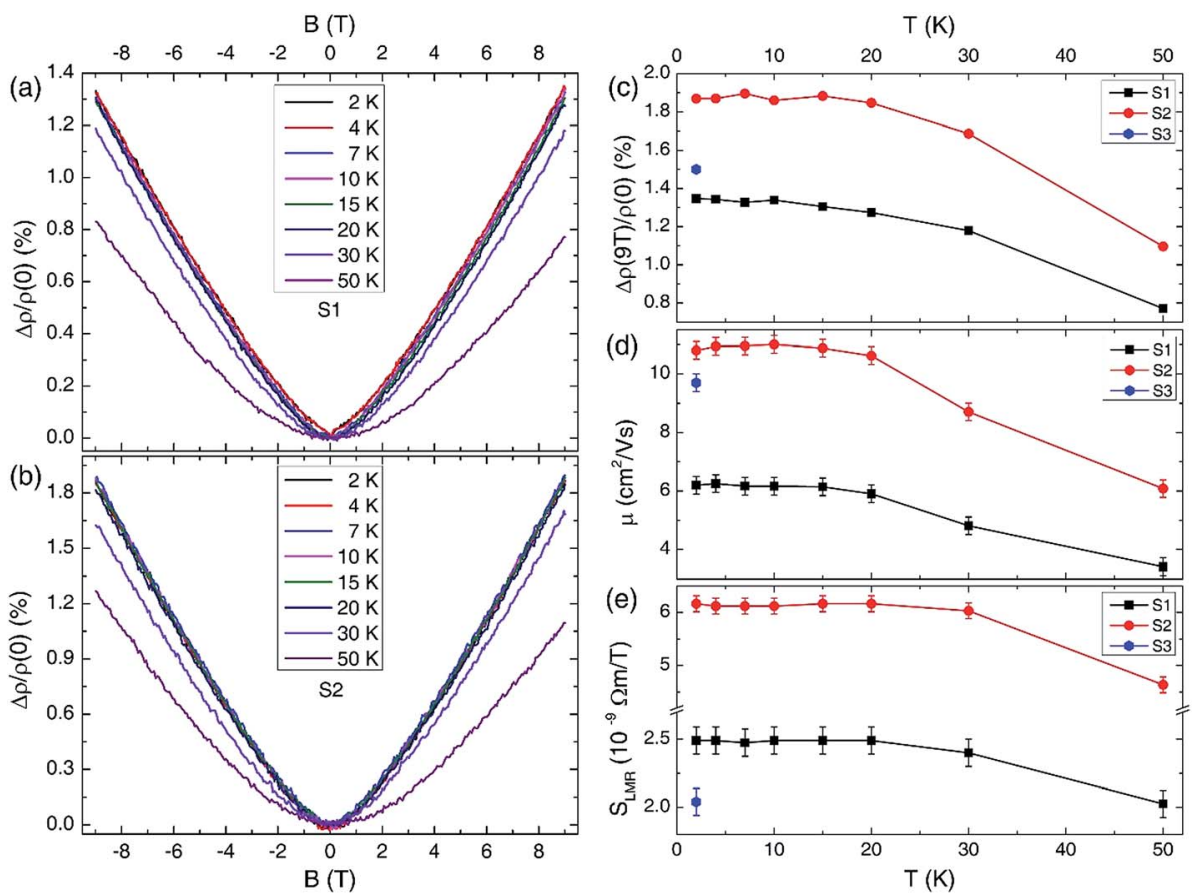

Fig. 3 Magnetotransport properties of the gold foams. (a) and (b) show the MR curves at several $T$ values as indicated for the foam samples S1 and S2, respectively. (c) Temperature dependence of $\Delta \rho(9 \mathrm{~T}) / \rho(0)$. (d) Temperature dependence of the electron mobility $\mu$ that is extracted by the Hall fitting. (e) Temperature dependence of the slope of the linear MR (LMR), that is, $S_{L M R} \equiv \mathrm{d} \rho(B) / d B$ at the high field regime.

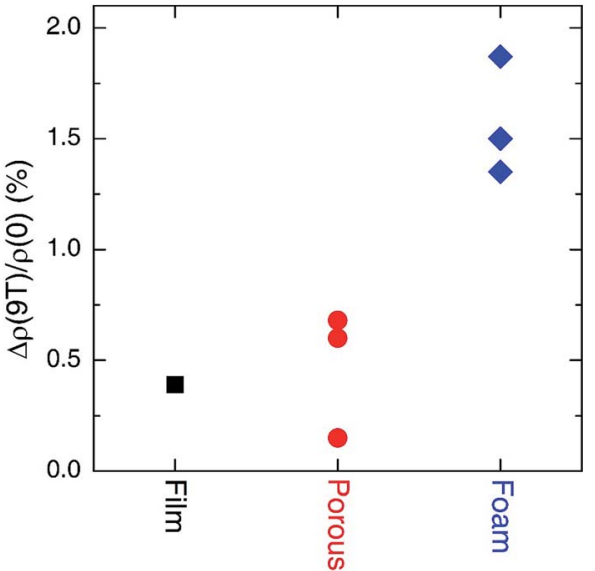

Fig. 4 Magnetoresistance values $\Delta \rho(9 \mathrm{~T}) / \rho(0)$ of the homogeneous gold films, the nanoporous gold films and the gold foams. The results of the nanoporous gold films are adapted from ref. 10.

Fig. 4 compares the MR values at a high magnetic field $(B=9$ T) for different forms of gold material. The MR values of the homogeneous gold films and the gold foams are shown (see Table 1 and Fig. 2). The MR values of the nanoporous gold at $B$ $=18 \mathrm{~T}$ and $T=4.2 \mathrm{~K}$ were adapted from the report by Fujita et $a l .{ }^{10}$ The typical value of $\Delta \rho(18 \mathrm{~T}) / \rho(0)$ for nanoporous gold is $0.6 \%$, which is larger than that of gold films but smaller than the $\Delta \rho(9 \mathrm{~T}) / \rho(0)$ values for our gold foams. The large difference of the MR values between the different gold forms further demonstrated that the electrical transport properties were affected by the 3D topology of the nanostructures.

\section{Discussions}

Now, we discuss the physical mechanisms behind the linear MR behavior in our gold foams. The linear MR phenomenon has been widely studied in several materials, such as $\mathrm{Ag}_{2 \pm \delta} \mathrm{Se},{ }^{\mathbf{1 8}}$ $\mathrm{Ag}_{2 \pm \delta} \mathrm{Te}^{19}{ }^{19} \mathrm{Bi}$ films, ${ }^{20} \mathrm{InSb},{ }^{21} \mathrm{Bi}_{2} \mathrm{Te}_{3},{ }^{22} \mathrm{Bi}_{2} \mathrm{Se}_{3},{ }^{23} \mathrm{Cd}_{3} \mathrm{As}_{2},{ }^{24} \mathrm{WTe}_{2},{ }^{25}$ TaAs, ${ }^{26} \quad \beta-\mathrm{RhSn}_{4},{ }^{27}$ GaAs quantum wells, ${ }^{28}$ graphene $^{29}$ and carbon foams. ${ }^{30-32}$ To the best of our knowledge, two types of theoretical models have been proposed to explain the linear MR phenomena: the classical model and the quantum model. One of the classical linear MR models was suggested by Parish and Littlewood (PL). ${ }^{33,34}$ They argued that the magnetotransport properties of the inhomogeneous materials were governed by the spatial mobility fluctuations rather than the mobility itself. The large mobility fluctuations would lead to a linear MR behavior in the strong disordered systems. Another classical model was proposed by Sampsell and Garland, ${ }^{35}$ and Stroud and Pan $^{36}$ (SGSP). They exactly calculated the electrical current distributions in the free-electron conductors with nonconducting spherical and cylindrical inclusions. The calculated results revealed that the current distortions occurred while the magnetic field was present. A larger field resulted in a larger current distortion. This effect led to a linear MR behavior in the high field regime. In contrast, a quantum model was developed by Abrikosov ${ }^{37}$ in 1969. According to Abrikosov's theory, a linear MR appeared in gapless semiconductors with a linear energy dispersion under the quantum limit condition, that is, the applied magnetic field must be so strong that only the lowest Landau level is populated. Recently, several quantum models have been proposed ${ }^{38,39}$ to interpret the linear MR 

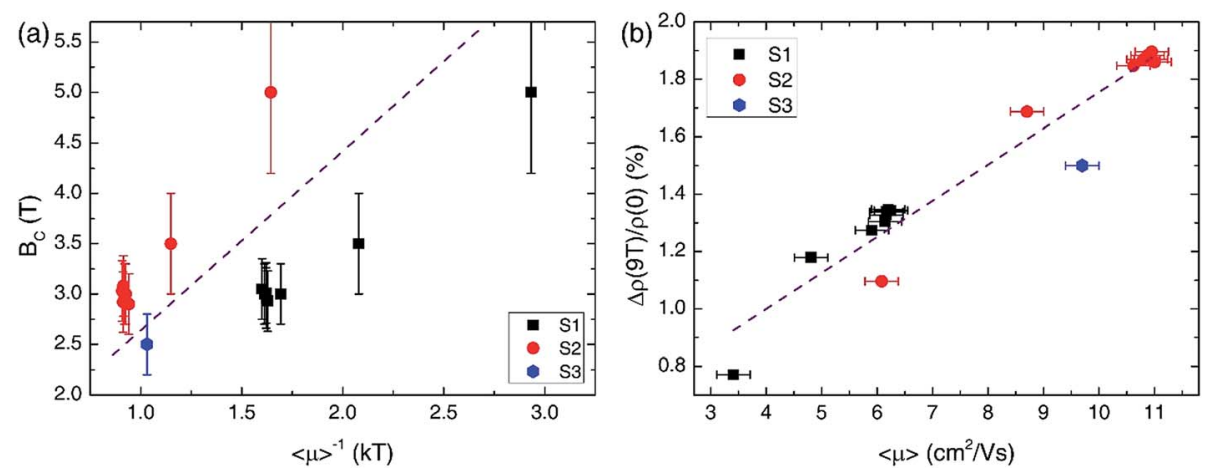

Fig. 5 Analysis of the PL theory. (a) Plot of the crossover field, $B_{C}$, versus the inverse of the average mobility, $\langle\mu\rangle^{-1}$. (b) $M R$ values $\Delta \rho(9 \mathrm{~T}) / \rho(0)$ plotted as a function of the average mobility $\langle\mu\rangle$. The dashed lines in (a) and (b) are used as guides for the eye.

properties in topological insulators ${ }^{22,23}$ and topological semimetals $^{24-26}$ that lie outside of the Abrikosov paradigm. These progresses were not discussed here as they are inappropriate for our foam materials.

In our samples, first we excluded Abrikosov's mechanism owing to the breaking of the quantum limit condition at such a low magnetic field $\left(E_{\mathrm{F}} / \hbar \omega_{\mathrm{C}} \sim 2500 \gg 1\right.$ at $B=9 \mathrm{~T}$, where $E_{\mathrm{F}}$ is the Fermi level and $\hbar \omega_{\mathrm{C}}$ is the first Landau level) in our gold foams. Next, we attempt to determine the applicability of the classical PL model in our gold foams. Obviously, the strong structure inhomogeneity of gold foams will leads to the spatial inhomogeneity of electron mobility. According to the PL theory, in the case of $\Delta \mu /\langle\mu\rangle<1$ (where $\langle\mu>$ is the average mobility and $\Delta \mu$ is the mobility fluctuation), we could expect (i) $B_{\mathrm{C}} \propto\langle\mu\rangle^{-1}$ ( $B_{\mathrm{C}}$ is the critical field above which the MR becomes linear) and (ii) $\Delta \rho / \rho(0) \propto\langle\mu\rangle$. Fig. 5(a) and (b) show the dependence of $B_{\mathrm{C}}$ on $\langle\mu\rangle^{-1}$ and the dependence of MR at $B=9 \mathrm{~T}$ on $\langle\mu\rangle$, respectively. These experimental results were consistent with the predictions from PL theory. However, the precondition of $\Delta \mu /<\mu><1$ deserves the experimental confirmation in the future. Finally, we considered the classical SGSP theory. This predicted a theoretical expression for the linear MR for samples containing spherical and cylindrical voids: $:^{35,36,40}$

$$
\frac{\mathrm{d} \rho(B)}{\mathrm{d} B}=\frac{\alpha f}{n_{0} e}
$$

where $n_{0}$ is the electron concentration of the matrix material, $e$ is the electron charge, $f$ is the volume fraction of the voids $(f \sim$ 0.8 for our gold foams), and $\alpha$ is a numerical constant that is $\alpha=$ 0.49 for spherical and $\alpha=1$ for cylindrical voids. We noted that the slope of the linear MR [i.e., $S_{\mathrm{LMR}} \equiv \mathrm{d} \rho(B) / \mathrm{d} B$ at the high field regime, see the insets of Fig. 2(a) and (b)] is temperature independent according to eqn (1). Fig. 3(e) displays the temperature dependence of $S_{\mathrm{LMR}}$ for the gold foams. We observed that $S_{\mathrm{LMR}}$ is nearly a constant in the temperature range of $T \lesssim 30 \mathrm{~K}$, and the suppressed $S_{\mathrm{LMR}}$ at $T=50 \mathrm{~K}$ may stem from the enhancement of the conventional $B^{2}$ dependent MR contributions. Quantitatively, we can estimate the electron concentration of the gold material in the foams $n_{0} \sim 10^{21} \mathrm{~cm}^{-3}$ by using eqn (1). This value seems smaller than the value of $n_{\text {foam }} /(1-f) \sim 10^{22}$ $\mathrm{cm}^{-3}$, which was extracted by Hall analysis. Overall, the linear
MR in the gold foams was caused by the classical mechanisms. Both the PL and SGSP theories can qualitatively interpret the experimental results, and the detailed mechanism deserves further experimental clarification.

\section{Conclusions}

We experimentally studied the magnetotransport properties of the gold foams that were prepared by a chemical templatedealloying method. The temperature dependence of the resistivity of the gold foams represents a metallic behavior with zero magnetic field. With an increasing magnetic field, a crossover from a quadratic to linear dependence of the magnetoresistance and the nonsaturating linear magnetoresistance in gold foams are observed. The physical mechanisms of the linear magnetoresistance in the gold foams are discussed within the frameworks of the classical PL and SGSP theories, but further investigations are required to determine the exact mechanism. Our work reveals a linear magnetoresistance phenomenon in the gold foams, which may be useful for future applications.

\section{Acknowledgements}

This work is financially supported by the National Natural Science Foundation of China (Grant No. 11604310, 11404304, 60908023), the National Key Scientific Instrument and Equipment Development Project of China (Grant No. 2014YQ090709) and the Key Laboratory of Ultra-Precision Machining Technology Foundation of CAEP (Grant No. ZZ15003).

\section{References}

1 H. L. Stormer, Rev. Mod. Phys., 1999, 71(4), 875.

2 F. A. Zwanenburg, A. S. Dzurak, A. Morello, et al., Rev. Mod. Phys., 2013, 85(3), 961.

3 A. G. Aronov and Y. V. Sharvin, Rev. Mod. Phys., 1987, 59(3), 755.

4 I. Zutic, J. Fabian and S. D. Sarma, Rev. Mod. Phys., 2004, 76(2), 323.

5 J.-C. Charlier, X. Blase and S. Roche, Rev. Mod. Phys., 2007, $79(2), 677$. 
6 S. D. Sarma, S. Adam, E. H. Hwang and E. Rossi, Rev. Mod. Phys., 2011, 83(2), 407.

7 M. Z. Hasan and C. L. Kane, Rev. Mod. Phys., 2010, 82(4), 3045.

8 H. Yu, X. Cui, X. Xu and W. Yao, Natl. Sci. Rev., 2015, 2(1), 57.

9 B. C. Tappan, S. A. Steiner-III and E. P. Luther, Angew. Chem., Int. Ed., 2010, 49(27), 4544.

10 T. Fujita, H. Okada, K. Koyama, K. Watanabe, S. Maekawa and M. W. Chen, Phys. Rev. Lett., 2008, 101(16), 166601.

11 R. Xia, J. L. Wang, R. Wang, et al., Nanotechnology, 2010, 21(8), 085703.

12 A. K. Mishra, C. Bansal and H. Hahn, J. Appl. Phys., 2008, 103(9), 094308.

13 P. Wahl, T. Traußnig, S. Landgraf, H.-J. Jin, J. Weissmuller and R. Wurschum, J. Appl. Phys., 2010, 108(7), 073706.

14 M. Hakamada, N. Kato and M. Mabuchi, Appl. Surf. Sci., 2016, 387, 1088.

15 K. Zhang, X. Tan, J. Zhang, W. Wu and Y. Tang, RSC Adv., 2014, 4(14), 7196.

16 J. S. Dugdale, The electrical properties of disordered metals, New York, Cambridge University Press, 1995.

17 C. Kittel, Introduction to Solid State Physics, New Jersey, John Wiley \& Sons Inc, 2005.

18 R. Xu, A. Husmann, T. F. Rosenbaum, M. L. Saboungi, J. E. Enderby and P. B. Littlewood, Nature, 1997, 390(6655), 57-60, DOI: 10.1038/36306.

19 M. Lee, T. F. Rosenbaum, M.-L. Saboungi and H. S. Schnyders, Phys. Rev. Lett., 2002, 88(6), 066602.

20 F. Y. Yang, K. Liu, K. Hong, D. H. Reich, P. C. Searson and C. L. Chien, Science, 1999, 284, 1335.

21 J. Hu and T. F. Rosenbaum, Nat. Mater., 2008, 7(9), 697-700, DOI: $10.1038 /$ nmat2259.

22 X. Wang, Y. Du, S. Dou and C. Zhang, Phys. Rev. Lett., 2012, 108(26), 266806.
23 H. T. He, H. C. Liu, B. K. Li, et al., Appl. Phys. Lett., 2013, 103(3), 031606.

24 T. Liang, Q. Gibson, M. N. Ali, M. Liu, R. J. Cava and N. P. Ong, Nat. Mater., 2015, 14, 280.

25 M. N. Ali, J. Xiong, S. Flynn, et al., Nature, 2014, 514, 205.

26 X. Huang, L. Zhao, Y. Long, et al., Phys. Rev. X, 2015, 5(3), 031023.

27 X. Z. Xing, C. Q. Xu, N. Zhou, et al., Appl. Phys. Lett., 2016, 109(12), 122403.

28 T. Khouri, U. Zeitler, C. Reichl, et al., Phys. Rev. Lett., 2016, $117(25), 256601$.

29 F. Kisslinger, C. Ott, C. Heide, et al., Nat. Phys., 2015, 11(8), 650.

30 L. Wang, M. Yin, M. Abdi and T. Datta, Appl. Phys. Lett., 2015, 107(2), 023103.

31 P. Li, Q. Zhang, X. He, W. Ren, H.-M. Cheng and X.-X. Zhang, Phys. Rev. B: Condens. Matter Mater. Phys., 2016, 94(4), 045402.

32 Y. Tanabe, Y. Ito, K. Sugawara, et al., Adv. Mater., 2016, 28(46), 10304.

33 M. M. Parish and P. B. Littlewood, Nature, 2003, 426, 162.

34 M. M. Parish and P. B. Littlewood, Phys. Rev. B: Condens. Matter Mater. Phys., 2005, 72(9), 094417.

35 J. B. Sampsell and J. C. Garland, Phys. Rev. B: Solid State, 1976, 13(2), 583.

36 D. Stroud and F. P. Pan, Phys. Rev. B: Solid State, 1976, 13(4), 1434.

37 A. A. Abrikosov, Soviet Physics - JETP, 1969, 29(4), 746.

38 C. M. Wang and X. L. Lei, Phys. Rev. B: Condens. Matter Mater. Phys., 2012, 86(3), 035442.

39 J. C. W. Song, G. Refael and P. A. Lee, Phys. Rev. B: Condens. Matter Mater. Phys., 2015, 92(18), 180204.

40 C. J. Beers, J. C. M. V. Dongen, H. V. Kempen and P. Wyder, Phys. Rev. Lett., 1978, 40(18), 1194. 